\title{
An Inorganic Thymidine Helix: Design of Homochiral Helical Arrays
}

Arne Roth, Daniel Koth, Michael Gottschaldt, and Winfried Plass

\section{Supplementary experimental data:}

General methods. Electronic spectra were recorded with a Varian Cary 1 or Cary 5E spectrophotometer at room temperature. IR spectra were measured on a Perkin-Elmer 2000 or a Bruker Equinox 55 spectrometer, NMR spectra on a Bruker AC-200, mass spectra were carried out on a Finnigan MAT SSQ 710 or a Finnigan MAT 95XL TRAP, elemental analyses on a Leco CHNS 932. Magnetic susceptibilities were measured on a MPMSR-5SSQUID magnetometer from Quantum Design from 2 to $300 \mathrm{~K}$ at magnetic fields of 2000 and 5000 Oe. Samples were placed in gelatine capsules and diamagnetic corrections were calculated according to Pascal's constants. Temperature-dependent X-band EPR spectra were recorded on a Bruker ESP 300 E spectrometer provided with a helium cryostate. Samples were measured as powder or as frozen methanolic solution.

X-ray crystallographic studies. The intensity data were collected on a Nonius KappaCCD diffractometer, using graphite-monochromated $\mathrm{Mo}_{\mathrm{\alpha}} \mathrm{K}_{\alpha}$ radiation. Data were corrected for Lorentz and polarization effects, but not for absorption. ${ }^{1-3}$ The structures were solved by direct methods $\left(S H E L X S^{4}\right)$ and refined by full-matrix least squares techniques against $\mathrm{F}_{0}{ }^{2}$ $\left(\right.$ SHELXTL97-2 $\left.{ }^{5}\right)$. All non-hydrogen atoms were refined anisotropically. ${ }^{4}$ The programs $X P$ (SIEMENS Analytical X-ray Instruments, Inc.) and Diamond ${ }^{6}$ were used for structure representations.

\section{Magnetic susceptibility measurements:}

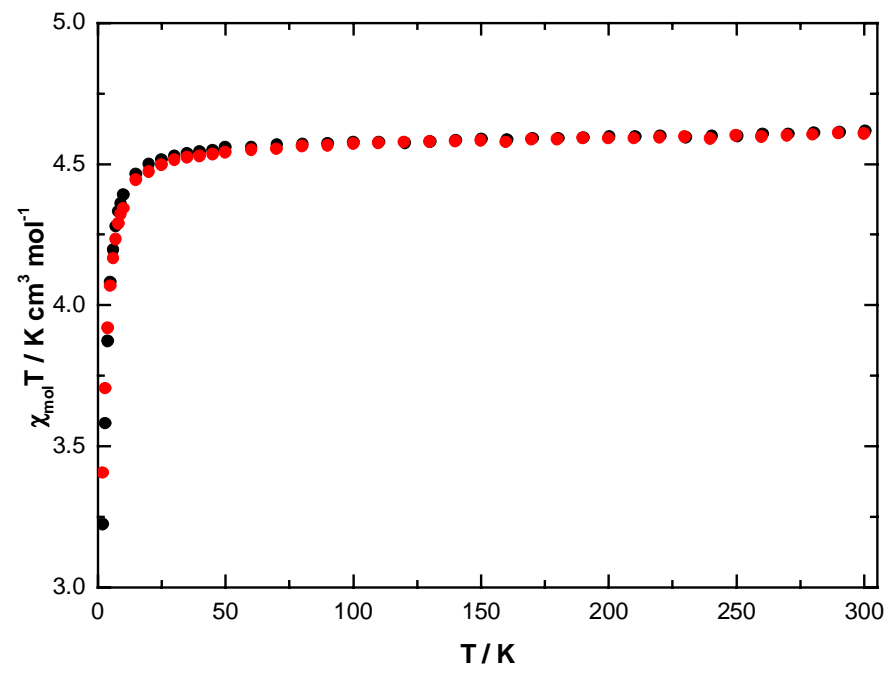


Figure S1: $\chi_{\mathrm{mol}} T$-plot of complex Fe1. The magnetic susceptibility was measured from 2 to $300 \mathrm{~K}$ at magnetic fields of 2000 (red) and $5000 \mathrm{Oe}$ (black).

\section{References}

[1] SMART COLLECT, Data Collection Software; Nonius B. V., Netherlands, 1998.

[2] Otwinowski, Z.; Minor, W. Processing of X-Ray Diffraction Data Collected in Oscillation Mode in Methods in Enzymology, Vol. 276, Macromolecular Crystallography, Part A, 307-326, Carter, C.W.; Sweet, R.M. (editors), Academic Press, 1997.

[3] SMART, Software for the CCD Detektor System, version 5.05: Bruker AXS: Madison, WI, 1998.

[4] Sheldrick, G.M. Acta Crystallogr. 1990, A46, 467-473.

[5] Sheldrick, G.M. SHELXTL97-2, University of Göttingen, Germany, 1997.

[6] Brandenburg, K. Diamond 3.1a, Crystal Impact GbR, Bonn, Germany, 1997-2005. 